\title{
Contrat agro-environnemental et participation des agriculteurs
}

The agroenvironmental contract and farmers' participation: A state of the art

\section{Olivier Carvin et Sandra Saïd}

\section{(2) OpenEdition}

1 Journals

Édition électronique

URL : https://journals.openedition.org/economierurale/8131

DOI : 10.4000/economierurale.8131

ISSN : 2105-2581

Éditeur

Société Française d'Économie Rurale (SFER)

Édition imprimée

Date de publication : 30 septembre 2020

Pagination : 95-109

ISSN : 0013-0559

\section{Référence électronique}

Olivier Carvin et Sandra Saïd, «Contrat agro-environnemental et participation des agriculteurs »,

Économie rurale [En ligne], 373 | Juillet-septembre, mis en ligne le 04 janvier 2022, consulté le 07 janvier 2022. URL : http://journals.openedition.org/economierurale/8131 ; DOI : https://doi.org/ 10.4000/economierurale.8131 


\title{
ÉTAT DE L'ART
}

\section{Contrat agro-environnemental et participation des agriculteurs}

\author{
Olivier CARVIN • Université Clermont Auvergne, AgroParisTech, INRAE, VetAgro Sup, \\ Territoires, F-63000 CLERMONT-FERRAND, France \\ Sandra SAÏD • Université Clermont Auvergne, AgroParisTech, INRAE, VetAgro Sup, \\ Territoires, F-63000 CLERMONT-FERRAND, France
}

\begin{abstract}
L'objectif de cet article est de proposer un état de l'art du rôle joué par la conception (design) du contrat agro-environnemental sur la participation des agriculteurs. La diversité des dispositifs en Europe et dans le monde conduit les auteurs à mettre l'accent sur la conception de ces contrats et son impact sur la participation selon quatre dimensions : maintien ou changement de pratiques, logique de moyens ou de résultats, paiement uniforme ou différencié entre les agriculteurs, paiement individuel ou collectif. L'état de l'art réalisé rappelle que les mesures visant le maintien de pratiques ne constituent pas une solution pour tous types d'enjeux et que les contrats potentiellement innovants n'ont pas un impact positif clair sur la participation des agriculteurs.
\end{abstract}

MOTS-CLÉS : économie agricole, environnement, mesures agro-environnementales, participation

\section{The agroenvironmental contract and farmers' participation: $A$ state of the art}

The purpose of this paper is to draw up a state of the art of the design of the agroenvironmental contract and its impact on farmers' participation. The diversity of arrangements in Europe and around the world leads us to focus on contract design and its impact on participation, based on four dimensions: maintaining or changing practices, action-oriented or result-oriented payment, uniform or differentiated payment between farmers, individual or collective payment. The state of the art recalls that measures aiming to maintain practices are not a solution to all issues. Furthermore, innovative measures do not have a clearly positive impact on farmers' participation. (JEL: Q18, Q57)

KEYWORDS: agricultural economics, environment, agroenvironmental measures, participation

C réée par le traité de Rome en 1957, puis mise en œuvre à partir de 1962, la Politique agricole commune (PAC) devait répondre à plusieurs objectifs, dont l'autosuffisance alimentaire et l'accroissement de la productivité. Cela s'est traduit par la promotion d'une intensification et une modernisation de la production agricole soutenues par des subventions et des aides indirectes. Cette intensification a entraîné une dégradation de l'environnement qui a été dénoncée par des organisations environnementales dès les années 1970. Afin de répondre à cette inquiétude croissante, mais aussi pour faire face aux critiques émises par l'Organisation mondiale du commerce (OMC) sur le subventionnement de l'agriculture, les Mesures agro-environnementales (MAE) ont été mises en place.

Les MAE sont des contrats de 5 ans, proposés à des agriculteurs volontaires. Ceux-ci s'engagent à maintenir ou adopter des pratiques agricoles respectueuses de l'environnement. En contrepartie, ils reçoivent une compensation financière annuelle correspondant aux coûts de mise en œuvre et aux pertes de revenus résultant de ces engagements. Les États membres de l'Union européenne (UE) ont l'obligation d'instaurer ces mesures depuis 1992 (règlement $n^{\circ}$ 2078/92).

L'évolution du dispositif français a permis un meilleur zonage des mesures, grâce 
à une mise en œuvre territorialisée (Gassiat et Zahm, 2013). Cependant, les MAE impliquant un réel changement de pratiques pour l'agriculteur, c'est-à-dire des investissements aussi bien en termes de capital qu'en termes de temps de travail, ont rencontré peu de succès auprès des agriculteurs. À l'inverse, les mesures visant un maintien de pratiques ont suscité un réel engouement. Par exemple, sur la période 2007-2013, $84 \%$ des mesures contractualisées dans des zones Natura 2000 sont des mesures de gestion extensive et visent un maintien des pratiques (MAAF, 2017). Le contraste entre le succès de ces mesures et le faible taux de contractualisation des mesures plus exigeantes nuance le bilan d'évaluation de la politique agroenvironnementale en France.

Un certain nombre de travaux portent sur les facteurs de participation ${ }^{1}$ des agriculteurs aux MAE, tels que les facteurs sociodémographiques, liés à l'exploitation, socio-institutionnels, comportementaux, et aux caractéristiques du contrat. Cette dernière catégorie de facteurs apparaît fortement déterminante quant à la participation des agriculteurs. De plus, la diversité des contrats agro-environnementaux mis en œuvre en Europe et dans le monde témoigne d'une grande hétérogénéité de conception.

Nous proposons de présenter, par conséquent, la conception (design) des contrats agro-environnementaux et son impact sur la participation des agriculteurs selon quatre dimensions qui, selon nous, définissent les grandes orientations du cahier des charges : (1) maintien ou changement de pratiques, (2) logique de moyens ou de résultats, (3) paiement uniforme ou différencié dans le temps et entre les agriculteurs, (4) paiement individuel ou collectif. Dans le cadre de cette revue de littérature,

1. Nous parlons ici de participation dans le sens de « souscription ». nous nous intéressons en particulier au faible taux de souscription aux MAE les plus exigeantes. Dans un premier temps, nous réaliserons un bref historique du dispositif agro-environnemental en France, puis nous présenterons les différentes catégories de facteurs de participation pour nous concentrer, en dernier lieu, sur le rôle joué par la conception du contrat agroenvironnemental.

\section{Historique des mesures agro-environnementales en France}

La mise en œuvre des mesures agro-environnementales par les États de l'Union européenne est obligatoire depuis 1992 (règlement 2078/92). En France, cette obligation s'est traduite par la mise en place d'Opérations locales agri-environnement (OLAE) et de Plans de développement durable (PDD). Les OLAE reposaient sur des cahiers des charges types par enjeu environnemental et définis au niveau national, tandis que les PDD consistaient en un projet d'exploitation répondant à une multiplicité d'enjeux. La mise en place de ces dispositifs est restée marginale, puisque les engagements OLAE ne concernaient en 1999 que $2 \%$ (900 000 ha) de la Surface agricole utile (SAU) (Urbano et Vollet, 2005) et les souscriptions des contrats PDD qu'environ 700 agriculteurs.

En parallèle, dès 1993, a été instaurée la Prime au maintien des systèmes d'élevage extensifs (PMSEE) dont le cahier des charges était défini au niveau national et visait le maintien des prairies et des espaces ouverts à gestion extensive, une utilisation réduite de pesticides, ainsi que le respect de seuils de chargement en bétail. Peu contraignant, ce dispositif connut rapidement un grand succès dès son instauration, puisque plus de $15 \%$ (117 000) des agriculteurs y participèrent pour 5,8 millions d'hectares engagés. La prime représenta alors à elle seule plus de $75 \%$ du budget de la politique agro-environnementale en 
France (Couvreur et al., 1999). La différence notable en termes de nombre d'agriculteurs engagés et d'hectares souscrits dans les deux dispositifs fait apparaître un fort contraste entre le succès de la PMSEE et le faible nombre d'exploitations engagées dans d'autres dispositifs (OLAE et PDD). Ce contraste persistera au fil des différents systèmes mis en place par la suite.

En 1999, dans le cadre de la réforme «Agenda 2000» et du premier Plan de développement rural national (PDRN), les dispositifs OLAE et PDD furent remplacés par les Contrats territoriaux d'exploitation (CTE), qui reprenaient la logique d'exploitation des PDD, tandis que la PMSEE fut reconduite. En apparence, ce nouveau dispositif fut un succès étant donné qu'en 2003, près de 50000 (12\%) agriculteurs ont contractualisé des CTE, sur une surface totale de 3,65 millions d'hectares (13,6 \% de la SAU nationale) (Urbano et Vollet, 2005). Cependant, plus de la moitié des CTE se sont limités à la seule mesure «gestion extensive par la fauche » dont le principe est similaire à la PMSEE. Sur la même période, cette dernière a concerné 69000 agriculteurs, soit environ $17 \%$ du nombre d'exploitations.

À partir de 2003, dans le cadre de la réforme Fischler, les CTE furent remplacés par les Contrats d'agriculture durable (CAD) dont l'objectif était d'accroître l'efficacité environnementale au moyen de trois principaux leviers: l'obligation d'inscrire un volet environnemental dans le cadre du CAD, un plafonnement des aides reçues par l'agriculteur, ainsi qu'un zonage par actions prioritaires. La PMSEE fut reconduite sous l'appellation «Prime herbagère agro-environnementale » (PHAE) et bénéficia d'une revalorisation moyenne de $70 \%$.

Dans le cadre de l'élaboration du Programme de développement rural hexagonal (PDRH), les CAD furent remplacés en 2007 par les Mesures agro-environnementales territorialisées (MAET). Mises en place selon des cahiers des charges définis en fonction des enjeux environnementaux du territoire, les MAET poursuivirent la logique de territorialisation entreprise avec les CAD. En effet, ce sont désormais des opérateurs agro-environnementaux (collectivités territoriales, Chambres d'agriculture, etc.) qui doivent monter et animer le projet agro-environnemental. Malgré cet effort de territorialisation, les MAET restèrent largement minoritaires parmi les contrats souscrits par les agriculteurs, puisqu'elles représentèrent seulement $25 \%$ des dossiers annuels, contre plus de $50 \%$ pour la seule prime à l'herbe (PHAE) pour la période 2007-13 (MAAF, 2017).

Dans le cadre du nouveau plan hexagonal (PDRH) pour la période 2014-2020, les MAET furent remplacées par les Mesures agro-environnementales et climatiques (MAEC). Mise à part l'introduction de mesures « systèmes » qui proposent un engagement global de l'exploitation au lieu de la souscription de parcelles singulières, le principe reste inchangé. Cependant, il est important de noter la suppression de la prime à l'herbe (PHAE) qui était fortement critiquée au niveau européen. Sa mise en place sur des zones d'agriculture intensive était très limitée et concernait massivement un maintien de pratiques dans les exploitations de zones de montagne. Elle fut cependant reconduite à la fois au travers de la revalorisation de l'Indemnité compensatoire de handicaps naturels (ICHN) et au travers de la création de la mesure «Systèmes herbagers et pastoraux » (SHP) dont le principe est équivalent à l'ex-prime à l'herbe ${ }^{2}$. Par ailleurs, le relevé de conclusions du Conseil supérieur

2. Respect d'un taux maximal de chargement animal ; non-retournement de la surface toujours en herbe et absence de traitement phytosanitaire sur cette même surface. 
d'orientation (CSO, 2014) note que les nouvelles MAEC «systèmes » proposent une distinction entre les mesures ayant pour objectif le maintien de pratiques et celles visant le changement de pratiques ${ }^{3}$.

Ce bref historique amène deux constats importants.

En premier lieu, malgré la volonté d'assouplir le dispositif par une décentralisation progressive, la problématique principale du dispositif agro-environnemental demeure inchangée : la participation des agriculteurs se concentre principalement sur les mesures de type « prime à l'herbe » (MAAF, 2017). Or il a été montré que ces mesures à cahier des charges nationaux, visant généralement un maintien de pratiques, ont un impact relativement négligeable sur l'environnement (Pywell et al., 2012). L'efficacité environnementale pourrait être améliorée par une plus grande participation des agriculteurs à des mesures plus exigeantes (Chabé-Ferret et Subervie, 2013).

En second lieu, la forte participation des agriculteurs à des mesures de maintien de pratiques souligne leur faible intérêt pour des mesures plus exigeantes, mais susceptibles d'être mieux rémunérées. Bien que les MAEC actuelles affichent des montants de compensation allant de 50 à $900 € /$ ha, la majorité des agriculteurs souscrivent des mesures rémunérées à hauteur de 60 à $200 € /$ ha, s'agissant principalement des mesures «Systèmes herbagers et pastoraux » (SHP) et « Systèmes de polycultureélevage » (SPE). Par exemple, en Nouvelle Aquitaine, $70 \%$ des surfaces engagées sur la période 2015-2018 le sont dans le cadre de ces deux mesures (Ouvrard, 2019).

3. Les MAEC SHP ou polycultures-élevage « herbivores » (SPE) consistent à maintenir les pratiques existantes, alors que la MAEC systèmes grandes cultures vise à accompagner le changement de pratiques.
Il apparaît donc que le montant de la compensation ne peut être considéré comme le seul facteur déterminant la participation des agriculteurs. Nous proposons par conséquent d'identifier, dans la littérature académique existante, les différentes catégories de facteurs susceptibles d'influer, négativement ou positivement, la participation des agriculteurs à des mesures impliquant un changement de pratiques. Nous mettons en particulier l'accent sur le rôle joué par la conception du contrat agro-environnemental.

\section{Les facteurs de participation aux mesures agro-environnementales}

La littérature abonde de travaux sur les facteurs de participation aux contrats agroenvironnementaux. Dans cette section seront présentées cinq catégories de facteurs : (1) facteurs sociodémographiques, (2) facteurs liés à l'exploitation, (3) facteurs sociaux et institutionnels, (4) facteurs comportementaux, (5) facteurs liés aux caractéristiques du contrat agro-environnemental. La revue de la littérature a été réalisée avec le moteur de recherche Web of Science, en faisant appel à la combinaison des mots clés suivants : «factor», «participation», «agri-environmental measure », «agri-environmental scheme ». Cette combinaison de mots clés nous permet de regrouper l'ensemble des publications étudiant des facteurs de participation à un dispositif agro-environnemental. Afin de nous concentrer sur des dispositifs et des contextes pouvant être comparables au dispositif agro-environnemental européen, nous avons restreint le champ d'application aux publications en économie parues depuis 1999 (année de création du second pilier de la PAC) traitant des mesures agroenvironnementales ou des paiements pour services environnementaux appliqués dans les pays développés. 


\section{Facteurs socio-démographiques}

Dans de très nombreuses études, l'âge est perçu comme un facteur défavorable à la participation de l'agriculteur (LastraBravo et al., 2015). Le changement de technique lié à l'adoption d'une MAE peut entraîner certains investissements que les agriculteurs plus âgés ne souhaitent pas engager, étant donné qu'ils ne travailleront pas suffisamment d'années pour obtenir un retour sur cet investissement (Lichtenberg, 2004).

Par ailleurs, la participation de l'agriculteur est positivement corrélée à son niveau d'éducation (Defrancesco et al., 2008). En effet, les compétences techniques permettent de réduire à la fois le coût du changement des pratiques et les risques induits par ce changement de techniques (Giannakopoulos et Damianos, 2002).

Le revenu du foyer est un autre facteur influençant la décision de participer aux MAE. Certaines études montrent que les agriculteurs aux revenus les plus faibles auront plutôt tendance à choisir la mesure «mise-en-jachère » comparativement à d'autres mesures qui sont plus contraignantes et surtout plus coûteuses (Murphy et al., 2011). Au contraire, d'autres études indiquent une corrélation négative. En effet, les agriculteurs ayant des faibles revenus ont par nature plus d'aversion au risque. Ils préfèrent contractualiser une ou plusieurs MAE qui leur procurent une source de revenu fixe et certaine pendant une période donnée (Beharry-Borg et al., 2013).

Enfin, la contractualisation d'une MAE lors d'un dispositif précédent augmente la probabilité d'un nouvel engagement (Menozzi et al., 2015). En effet, la contractualisation d'une MAE pourrait engendrer des coûts fixes liés par exemple à un investissement ou à l'acquisition d'informations. Ces coûts seraient faibles pour un agriculteur ayant précédemment souscrit une MAE et seraient élevés pour un agriculteur «nouvel entrant» dans un dispositif agro-environnemental.

\section{Facteurs liés à l'exploitation}

La part des revenus du foyer liée à l'exploitation agricole constitue un facteur pertinent. De nombreuses études montrent que plus la part des revenus de l'exploitation agricole dans les revenus du foyer est élevée, plus la participation de l'agriculteur est faible (Unay Gailhard et Bojnec, 2015). En effet, la dépendance aux revenus agricoles accroît le risque lié à un changement de techniques, particulièrement pour les moyennes et grandes exploitations.

Par ailleurs, le degré d'extensification de l'exploitation est perçu comme étant un facteur significatif de participation aux MAE. Notamment, il a été montré que la part d'herbages permanents dans l'exploitation a un impact positif sur la participation de l'agriculteur (Frondel et al., 2012). Ce résultat peut s'expliquer par le fait que les mesures de gestion extensive constituent, pour ces exploitations, des mesures à maintien de pratiques et sont donc peu contraignantes.

De même, la revue de littérature sur le sujet révèle généralement un impact positif de la taille de l'exploitation sur la participation de l'agriculteur. En effet, une exploitation de plus grande surface, toutes choses égales par ailleurs, possède une plus grande capacité de résilience face au risque. Ainsi, un agriculteur ayant une grande exploitation sera moins sensible au risque qu'un propriétaire d'une petite exploitation (Allaire et al., 2009).

\section{Facteurs institutionnels}

L'appartenance à un groupement d'agriculteurs peut être un levier ou un frein à la souscription d'une MAE par le transfert formel ou informel de connaissances qui est développé au sein du groupe (Polman et Slangen, 2008). De nombreuses études ont montré que les groupes sociaux ont 
une influence significative sur la participation des agriculteurs, que cette influence soit positive (Barreiro-Hurlé et al., 2010) ou négative (Mettepenningen et al., 2011), selon l'environnement pris en compte. Par exemple, Brun et Chabé-Ferret (2014) ont montré que le syndicat agricole FNSEA influence la participation des agriculteurs envers les MAE les moins exigeantes sur le plan environnemental, comparativement aux autres syndicats agricoles tels que la Confédération paysanne et le MODEF qui, a priori, soutiennent les mesures les plus exigeantes.

La confiance sociale, c'est-à-dire la confiance envers le comportement des autres agriculteurs, permet aussi d'accroître largement la participation des agriculteurs dans le cas où un système d'amende pour non-respect du contrat est instauré. Ce résultat «suggère que la confiance sociale renforce l'effet incitatif du système punitif en encourageant les comportements similaires entre agriculteurs » (Alló et al., 2015).

Les agriculteurs sont aussi demandeurs d'un appui technique lorsqu'ils contractualisent une MAE (Espinosa-Goded et al., 2010), car les conseils techniques peuvent réduire considérablement les coûts liés à la recherche d'informations par les agriculteurs. La mise en place d'un appui technique rassure les agriculteurs sur leur choix et diminue une partie des coûts de mise en œuvre des nouvelles pratiques.

\section{Facteurs comportementaux}

Selon la théorie du comportement planifié, la décision de l'agriculteur de s'engager dans des pratiques plus respectueuses de l'environnement est influencée par l'attitude, les normes perçues (qui correspondent à la perception des facteurs sociaux et institutionnels décrits précédemment) et la capacité pressentie à réussir cet engagement (Ajzen, 1991). De nombreuses études ont analysé la participation des agriculteurs à travers le prisme de ce modèle (Menozzi et al., 2015).

L'attitude d'un individu envers un comportement correspond à l'évaluation, positive ou négative, qu'il fait de ce comportement. Dans le cadre des MAE, l'attitude de l'agriculteur est multiple. Il peut s'agir à la fois de l'évaluation de l'agriculteur envers la protection de l'environnement, envers l'impact de ses pratiques sur l'environnement, envers le dispositif agroenvironnemental, ou bien encore envers les pratiques auxquelles l'agriculteur doit s'engager dans le cadre de ce dispositif. Il a été montré, notamment, que l'attitude de l'agriculteur envers l'environnement a un impact positif sur sa volonté de souscrire à des MAE (Ma et al., 2012).

La capacité perçue à réussir l'engagement a plusieurs dimensions. Elle recouvre à la fois la perception de l'agriculteur concernant ses connaissances et compétences techniques, et le comportement de l'agriculteur face au risque, puisque tout changement de pratiques comporte un risque. Par exemple, plus l'aversion au risque d'un agriculteur est importante, plus sa volonté de changer ses pratiques vers celles plus respectueuses de l'environnement sera faible (Giovanopoulou et al., 2011).

\section{Les facteurs liés aux caractéristiques du contrat agro-environnemental}

Un contrat agro-environnemental prend la forme d'un cahier des charges déclinant entre autres les engagements souscrits par l'agriculteur, la durée du contrat, les modalités du contrôle et le montant de la prime. Plusieurs études ont montré qu'un changement dans l'une de ces caractéristiques était susceptible d'améliorer le taux de contractualisation des agriculteurs. Un résultat admis est l'impact positif du montant de la compensation sur la participation de l'agriculteur, quels que soient le pays ou le mode d'agriculture concernés (Kuhfuss 
et al., 2014). Ainsi, une mesure peut avoir un succès particulièrement élevé par rapport à d'autres mesures dans une même région, parce que les agriculteurs considèrent que le paiement couvre totalement leurs coûts d'adaptation (Keenleyside et al., 2011). Par ailleurs, une réduction de la durée du contrat ou la possibilité de se retirer à tout moment du contrat, permettrait une augmentation de la participation des agriculteurs (Christensen et al., 2011).

Enfin, les agriculteurs sont aussi sensibles aux procédures de contrôles. Ainsi, l'augmentation de la probabilité que l'agriculteur soit contrôlé impacte négativement la participation (Broch et Vedel, 2012). En outre, les agriculteurs ont une préférence pour un contrôle annuel plutôt qu'un contrôle aléatoire (Peterson, 2011). De même, l'instauration d'une amende dans le cas où l'agriculteur contrôlé n'est pas en conformité avec les engagements pris est généralement perçue négativement par les agriculteurs (Alló et al., 2015).

Les caractéristiques du contrat, telles que le montant et la durée, ont donc bien un impact sur la participation des agriculteurs. Nous mettons l'accent sur cette catégorie de facteurs, puisque c'est sur ces caractéristiques que le décideur public peut avoir une influence directe afin d'améliorer le taux de participation des agriculteurs. Dans la section qui suit, nous proposons d'analyser en détail la conception du contrat agro-environnemental, c'est-à-dire les grandes orientations qui définissent le cahier des charges du contrat.

\section{La conception du contrat agro-environnemental}

Les différents contrats agro-environnementaux qui ont été mis en œuvre en Europe ou dans le monde témoignent d'une grande diversité. Pour avoir une idée plus précise de l'impact de la conception du contrat sur la participation des agriculteurs, nous proposons une revue plus ciblée de la littérature sur la conception des contrats agroenvironnementaux, selon quatre dimensions :

- «Maintien ou changement de pratiques », en fonction de l'objectif du cahier des charges par rapport aux pratiques agricoles déjà existantes.

- « Moyens ou résultats », en fonction du calcul de paiement reçu en contrepartie de la mise en œuvre du cahier des charges.

- «Uniforme ou différencié », en fonction de la variabilité du paiement dans le temps et entre les agriculteurs.

- «Individuel ou collectif», en fonction du destinataire du paiement.

Ainsi, un certain nombre de contrats potentiellement innovants ont été étudiés en détaillant les éventuels effets sur la participation.

\section{Maintien ou changement de pratiques}

À la lecture de l'article 28 du Règlement de développement rural (RDR 3), les MAEC spécifient distinctement, en termes de paiement et de cahier des charges, si l'objectif visé est le maintien ou l'évolution des pratiques agricoles. Les mesures visant un maintien de pratiques sont associées à celles de gestion extensive peu coûteuses en investissements et bénéficiant déjà d'un large succès auprès des agriculteurs. La problématique du faible taux de participation ne concerne donc pas ce type de mesures.

Par ailleurs, il ne nous semble pas cohérent de mettre en place des mesures se limitant au maintien de pratiques sur des zones où la qualité de l'environnement est fortement dégradée. Prenons l'exemple de la Directive-cadre sur l'eau (DCE) : elle pose comme objectif l'atteinte d'un bon état écologique de l'ensemble des masses d'eau de surface et souterraines. L'agriculture représente plus de $50 \%$ 
des rejets azotés dans les eaux de surface (European Commission, 2010), particulièrement en France où la pollution due aux nitrates demeure élevée (Cour des comptes, 2010). L'amélioration de la qualité des masses d'eau ne peut être atteinte sans réduction considérable des pesticides utilisés. Cependant, cette diminution est généralement synonyme de baisse de productivité qui se répercute par un manque à gagner pouvant être compensé par l'économie réalisée au niveau des quantités d'intrants achetées, ainsi qu'en termes de temps de travail et de carburant nécessaire aux machines d'épandage (Jacquet et al., 2011). Par ailleurs, la décision de participer à ce type de mesures conduit l'agriculteur à tenir compte de plusieurs autres éléments tels que l'incertitude environnementale et climatique, ainsi que son aversion au risque (Dessart et al., 2019). Comme indiqué déjà plus haut, la décision d'engagement de l'agriculteur dans un changement de pratiques peut en outre être influencée par une précédente expérience d'adhésion à des mesures visant le maintien de pratiques. Ces dernières sont perçues positivement par les agriculteurs, qui considèrent qu'elles valorisent leurs pratiques (Escot et al., 2013), tout en favorisant une plus grande prise de conscience concernant le caractère multifonctionnel de l'agriculture et son impact sur l'environnement (Menozzi et al., 2015).

\section{Logique de moyens ou de résultats}

En Europe, les paiements agroenvironnementaux se font principalement au titre des pratiques agricoles à adopter ou conserver. Par conséquent, le paiement est établi comme une compensation des coûts additionnels supportés, des pertes potentielles de revenus et des coûts administratifs qui sont intégrés à un maximum de $20 \%$ de la rémunération totale. Cependant, le cahier des charges imposé est parfois perçu, par les agriculteurs, déconnecté de la réalité du terrain, ce qui peut avoir un impact sur la participation de ces derniers (Kuhfuss et al., 2012).

Un paiement en fonction des résultats environnementaux augmente l'intérêt personnel de l'agriculteur en lui laissant le choix des pratiques à mettre en place pour atteindre l'objectif environnemental fixé par le décideur (McGurn et Moran, 2013). Ce principe, dont la mise en place est encouragée par la Cour des comptes européenne (2011), existe déjà en France depuis 2007, au travers du dispositif « Prairies Fleuries $»^{4}$ (Fleury et al., 2015). Néanmoins, l'agriculteur n'est pas totalement libre de ses pratiques dans ce cadre, puisqu'il doit respecter un taux de fertilisation maximum. Des paiements similaires existent dans d'autres pays d'Europe tels que la Suisse (l'Ökoqualitätsverodnung) et l'Allemagne (le programme MEKA dans le Bade-Wurtemberg) (Schwarz et Morkvenas, 2013). Ce principe de paiement semble être un succès sur le plan environnemental (Knop et al., 2006).

Par ailleurs, la littérature relève un certain nombre d'avantages à la mise en place de ce type de mesures pour l'agriculteur (Herzon et al., 2018). Notamment, son implication est accrue en comparaison à un paiement basé sur une logique de moyens, ce qui peut avoir un impact positif sur ses considérations environnementales. De plus, les pratiques qu'il adopte afin d'atteindre le résultat environnemental fixé sont supposément plus adaptées aux spécificités de l'exploitation qu'une mesure uniformisée et basée sur une logique de moyens (Birge et al., 2017). Ce type de paiement semble donc avoir souvent la préférence des agriculteurs (Wezel et al., 2018).

Néanmoins, les paiements basés sur une logique de résultats ne constituent

4. L'agriculteur s'engage à avoir, sur la parcelle engagée, au moins quatre espèces de plantes indicatrices d'un haut niveau de biodiversité. 
pas nécessairement une solution adéquate à tout enjeu environnemental. En effet, le transfert monétaire est conditionné à l'aboutissement d'un gain environnemental, lequel reste soumis aux aléas climatiques et aux effets de seuil. Les MAE ayant une logique de moyens assurent donc un revenu plus certain que celles basées sur une logique de résultats. Par ailleurs, pour une mise en œuvre réussie, ces dernières doivent être associées à un indicateur de performance environnemental crédible, facilement quantifiable et compréhensible par les institutions et les agriculteurs (Burton et Schwarz, 2013).

\section{Paiement uniforme ou différencié}

En France, les paiements sont uniformes par hectare et par action environnementale. Bien qu'il existe une adaptation régionale, ce dispositif généralisé entraîne un risque important de sélection adverse (Chabé-Ferret et Subervie, 2013). En effet, la contractualisation se heurte au problème d'asymétrie d'information entre le décideur et les agriculteurs. Contrairement au premier, les derniers connaissent leurs vrais coûts d'adoption. Cela implique des situations de surcompensation ou de souscompensation, étant donné que le paiement reçu est calculé sur une estimation des coûts moyens de mise en œuvre au niveau régional.

D'autres pays choisissent un type de paiement plus flexible, en fonction des critères spécifiques. Par exemple, la BasseAutriche établit un système d'éco-points ${ }^{5}$ (Gault et al., 2013); l'Italie élabore un paiement dépendant du relief géographique $^{6}$; la Pologne met en place une rémunération, dont le montant du paiement

5. Le système d'éco-points évalue l'intensité et la justesse de l'utilisation des ressources vis-à-vis de l'environnement.

6. Afin de prendre en compte les difficultés rencontrées par les agriculteurs en zone de montagne. par hectare est fonction de la taille de la surface souscrite (IEEP, 2011).

Un mécanisme d'allocation des contrats agro-environnementaux alternatif, appelé enchère agro-environnementale, permet d'aboutir à un type de paiement encore plus différencié (Thoyer et Saïd, 2007). L'enchère est utilisée notamment en Australie dans le cadre du programme Bush Tender (Stoneham et al., 2003) et aux États-Unis dans le cadre du Conservation Reserve Program et EQIP (Vukina et al., 2008). Basée sur la concurrence et la sélection des agriculteurs, elle peut ainsi donner lieu à un type de paiement différencié : deux agriculteurs appliquant le même cahier des charges ne perçoivent pas le même montant de prime. Si une telle mise en concurrence peut être acceptée dans les pays anglophones, en France, le principe d'un paiement différencié pour des résultats environnementaux identiques est susceptible d'être mal accepté (Kuhfuss, 2013).

\section{Paiement individuel ou collectif}

La PAC 2014-2020 prévoit la possibilité de rémunérer les actions collectives d'agriculteurs, sous réserve que les coûts de coordination induits par l'action collective soient

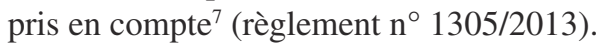
Aux Pays-Bas, il existe de nombreux groupements d'agriculteurs appelés « coopératives environnementales » qui, jusqu'en 2003, percevaient directement un paiement collectif sous contrainte d'avoir une surface minimale engagée. Elles étaient alors chargées du contrôle, ainsi que de la redistribution du paiement aux agriculteurs adhérents, sans qu'aucune règle concernant les modalités de cette redistribution ne leur soit imposée (Franks et McGloin, 2007 ; Gault et al., 2013). Depuis 2003, les agriculteurs sont payés individuellement,

7. Coûts estimés à hauteur de $30 \%$ du paiement total dans le cas d'actions collectives. 
mais les coopératives reçoivent un paiement compensant les frais de coordination (Gault et al., 2013). Actuellement, au sein de l'Union européenne, seuls les Pays-Bas ont réglementé la possibilité de mettre en ouvre des contrats collectifs. Or ce type d'approche collective a permis d'accroître la participation des petits exploitants (Franks et McGloin, 2007) pour lesquels les coûts d'entrée sont trop élevés lorsque le paiement est individuel. Les contrats collectifs représentent ainsi $39 \%$ de la SAU totale sous contrats agroenvironnementaux aux Pays-Bas (Van Dijk et al., 2015). Par ailleurs, par l'effet d'entraînement qu'il implique (Kuhfuss et al., 2016), un paiement collectif peut réduire le taux de non-renouvellement de contrat des agriculteurs.

Cependant, ce système peut fonctionner seulement si les groupements ont une marge de manœuvre suffisante dans la construction du cahier des charges. Il peut également impliquer des coûts de mise en œuvre et de coordination plus importants pour les groupements qui, à terme, peuvent impacter la participation des agriculteurs (Jongeneel et al., 2008). Ainsi, les contrats collectifs fonctionnent aux Pays-Bas, car ces coopératives environnementales sont présentes depuis plusieurs décennies, mais des enquêtes ont montré que ce même système n'est pas facilement adaptable à tout territoire (Riley et al., 2018).

Une conception des contrats agro-environnementaux basée sur le maintien de pratiques bénéficie d'un large succès auprès des agriculteurs, bien qu'elle ne soit pas adaptée à tous les enjeux environnementaux. Cependant, elle pourrait être une étape préalable à la mise en place ultérieure d'une conception basée sur un changement de pratiques pour contractualiser des mesures exigeantes.

Les contrats à logique de résultats doivent être privilégiés dans les cas où le gain environnemental est peu impacté par les conditions climatiques ou par les pratiques des autres agriculteurs ${ }^{8}$. Cette conception de contrat laisse à l'agriculteur la liberté de mettre en place les pratiques agricoles souhaitées à condition d'atteindre l'objectif environnemental fixé. Une conception basée sur un paiement différencié dans le temps, tel un paiement dégressif, pourrait favoriser la décision de participation à des mesures plus exigeantes. Enfin, un contrat avec paiement collectif peut favoriser la participation des agriculteurs, lorsque ceux-ci sont déjà coordonnés autour d'autres projets de territoire. Par exemple, cela peut être l'existence d'un groupement d'intérêt économique et environnemental (GIEE) qui rassemble les agriculteurs autour d'objectifs environnementaux communs.

Ainsi, face à l'hétérogénéité des territoires et des enjeux environnementaux existants, il est intéressant de mettre l'accent sur la conception des contrats agroenvironnementaux pour construire des dispositifs originaux en France. Un contrat combinant à la fois une logique de résultats avec un paiement différencié - ou un paiement collectif avec un changement de pratiques - pourrait convaincre les agriculteurs de participer à des mesures plus exigeantes. Ce type de contrat pourrait être plus en phase avec les nouvelles attentes de la société en termes de fourniture de services environnementaux.

$$
\begin{gathered}
* \\
* \quad *
\end{gathered}
$$

Depuis sa mise en place en 1992, le dispositif agro-environnemental est caractérisé par la coexistence entre, d'une part, des mesures de gestion extensive peu exigeantes et connaissant un large succès et, d'autre part, des mesures plus exigeantes qui ne bénéficient que d'un faible taux de participation. Le faible engagement dans

8. Par exemple, le cas de la diversité florale des prairies. 
ces mesures limite l'efficacité environnementale et pose la question légitime du lien entre la conception du contrat et la participation. En effet, en France, quel que soit le contrat, il est souvent conçu en fonction d'une logique de moyens pour calculer un paiement uniforme à destination individuelle.

L'objectif de ce travail a été de dresser un état de l'art sur le lien entre la participation des agriculteurs et la conception des contrats, en proposant une typologie selon quatre dimensions. Si un contrat visant le maintien des pratiques est largement préféré par les agriculteurs, il ne constitue cependant pas une solution adéquate à tous les enjeux environnementaux. Les contrats à objectifs de résultats sont principalement employés pour des mesures visant le maintien de pratiques. Leur faisabilité et leur acceptabilité pour d'autres types de mesures restent encore à démontrer. Une mesure intégrant un paiement différencié semble être une solution intéressante, mais peu d'études ont montré l'intérêt des agriculteurs pour ce type de mesures en France. Enfin, l'instauration d'un paiement collectif peut avoir un impact positif sur la participation à condition que les coûts de coordination ne soient pas trop élevés.

Notre analyse met cependant en lumière un besoin de recherches sur l'impact de ces alternatives en termes de participation selon le type de mesure ciblée (enjeu environnemental ciblé, niveau de contraintes, etc.) et les caractéristiques du territoire concerné (caractéristiques des exploitations, dynamique du territoire, etc.). Dans cette optique, les approches expérimentales d'évaluation sont un des outils pour répondre à ces questions (Thoyer et Preget, 2019). Les méthodes d'expérimentation des choix (choice experiment), par exemple, en révélant les préférences des agriculteurs en termes de caractéristiques du contrat agro-environnemental, permettent de déterminer sous quelles conditions une modification dans la conception du contrat agro-environnemental est favorable à la participation des agriculteurs (Latacz-Lohmann et Breustedt, 2019).

Ces recherches sont d'autant plus cruciales à l'heure où se construit la PAC 2021-2027. La reconduction des MAEC, telles qu'elles ont été conçues dans le dispositif actuel, semble être la solution privilégiée par la Commission européenne. Néanmoins, les MAEC pourraient être accompagnées d'un système « eco-scheme » qui bénéficierait d'une plus grande souplesse dans sa conception (European Commission, 2017). Il serait alors opportun de s'appuyer sur les divers enseignements tirés de la littérature traitée ici afin de mettre en œuvre un dispositif qui incite les agriculteurs à modifier durablement leurs pratiques. 


\section{RÉFÉRENCES BIBLIOGRAPHIQUES}

Ajzen I. (1991). The theory of planned behavior. Organizational Behavior and Human Decision Processes, Theories of Cognitive Self-Regulation, $\mathrm{n}^{\circ}$ 50, pp. 179-211, https:// doi.org/10.1016/0749-5978(91)90020-T.

Allaire G., Cahuzac E., Simioni M. (2009). Contractualisation et diffusion spatiale des mesures agro-environnementales herbagères. Revue d'études en agriculture et environnement, $\mathrm{n}^{\circ}$ 90, pp. 23-50.

Alló M., Loureiro M. L., Iglesias E. (2015). Farmers' Preferences and Social Capital Regarding Agri-environmental Schemes to Protect Birds. Journal of Agricultural Economics, $\mathrm{n}^{\circ} 66$, pp. 672-689, https://doi. org/10.1111/1477-9552.12104.

Barreiro-Hurlé J., $\quad$ Espinosa-Goded M., Dupraz P. (2010). Does intensity of change matter? Factors affecting adoption of agrienvironmental schemes in Spain. Journal of Environmental Planning and Management, $\mathrm{n}^{\circ} 53$, pp. 891-905, https://doi.org/10.1080/ 09640568.2010.490058

Beharry-Borg N., Smart J. C. R., Termansen M., Hubacek K. (2013). Evaluating farmers' likely participation in a payment programme for water quality protection in the UK uplands. Regional Environmental Change, $\mathrm{n}^{\circ} 13$, pp. 633-647, https://doi.org/10.1007/ s10113-012-0282-9.

Birge T., Toivonen M., Kaljonen M., Herzon I. (2017). Probing the grounds: Developing a payment-by-results agri-environment scheme in Finland. Land Use Policy, $\mathrm{n}^{\circ} 61$, pp. 302-315, https://doi.org/10.1016/ j.landusepol.2016.11.028.

Broch S. W., Vedel S. E. (2012). Using Choice Experiments to Investigate the Policy Relevance of Heterogeneity in Farmer Agri-Environmental Contract Preferences. Environmental and Resource Economics, $\mathrm{n}^{\circ} 51$, pp. 561-581, https://doi.org/10.1007/ s10640-011-9512-8.

Brun F., Chabé-Ferret S. (2014). Le rôle de l'orientation syndicale des Chambres d'agriculture dans la contractualisation des mesures agro-environnementales. VertigO, la revue électronique en sciences de l'environnement, https://doi.org/10.4000/vertigo.15292.

Burton R. J. F., Schwarz G. (2013). Resultoriented agri-environmental schemes in Europe and their potential for promoting behavioural change. Land Use Policy, $\mathrm{n}^{\circ} 30$, pp. 628-641, https://doi.org/10.1016/ j.landusepol.2012.05.002.

Chabé-Ferret S., Subervie J. (2013). How much green for the buck? Estimating additional and windfall effects of French agroenvironmental schemes by DID-matching. Journal of Environmental Economics and Management, $\mathrm{n}^{\circ}$ 65, pp. 12-27, https://doi. org/10.1016/j.jeem.2012.09.003.

Christensen T., Pedersen A. B., Nielsen H. O., Mørkbak M. R., Hasler B., Denver S. (2011). Determinants of farmers' willingness to participate in subsidy schemes for pesticide-free buffer zones-A choice experiment study. Ecological Economics, ${ }^{\circ} 70$, pp. 1558-1564. https://doi.org/10.1016/j. ecolecon.2011.03.021.

Cour des comptes (2010). Les instruments de la gestion durable de l'eau. Rapport public annuel-2010, pp. 617-655.

Cour des Comptes Européennes (2011). L'aide agro-environnementale est-elle conçue et gérée de manière satisfaisante? Rapport Spécial $\mathrm{n}^{\circ} 7$, Luxembourg, Cour des Comptes européennes, $75 \mathrm{p}$.

Couvreur M., Mitteault F., Pech M. (1999). Les mesures agri-environnementales mises en œuvre en France. Économie rurale, $\mathrm{n}^{\circ} 249$, pp. 6-10, https://doi.org/10.3406/ ecoru.1999.5053.

CSO (2014). PAC2014/2020 - Application en France Relevé de conclusions suite au CSO du 27 mai 2014, https://agriculture.gouv.fr/ sites/minagri/files/documents//Document_ CSO_27_mai_2014_-_releve_de_conclusions_cle877788.pdf.

Defrancesco E., Gatto P., Runge F., Trestini S. (2008). Factors Affecting Farmers' Participation in Agri-environmental Measures: A Northern Italian Perspective. Journal of Agricultural Economics, $\mathrm{n}^{\circ}$ 59, 
pp. 114-131, https://doi.org/10.1111/j.14779552.2007.00134.x.

Dessart F. J., Barreiro-Hurlé J., van Bavel R. (2019). Behavioural factors affecting the adoption of sustainable farming practices: a policy-oriented review. European Review of Agricultural Economics, $\mathrm{n}^{\circ}$ 46, pp. 417-471, https://doi.org/10.1093/erae/jbz019.

Escot E., Grandmougin B., Rossi A., Isel S., Barros C., Demouy Y., Wilmes C. (2013). Evaluation de la mise en æuvre des Mesures agro-environnementales en Alsace dans une optique de réforme de la PAC post 2013. RRR Alsace Éditions, 117 p.

Espinosa-Goded M., Barreiro-Hurlé J., Ruto E. (2010). What Do Farmers Want From AgriEnvironmental Scheme Design? A Choice Experiment Approach. Journal of Agricultural Economics, $\mathrm{n}^{\circ}$ 61, 259-273, https://doi. org/10.1111/j.1477-9552.2010.00244.x.

European Commission (2010). Commission Staff Working Document On implementation of Council Directive 91/676/EEC concerning the protection of waters against pollution caused by nitrates from agricultural sources based on Member State reports for the period 2004-2007. Accompanying document to the Report from the Commission to the Council and the European Parliament COM (2010) 47, 41 p.

European Commission (2017). Communication from the Commission to the European Parliament, the Council, the European Economic and Social Committee and the Committee of the Regions. The Future of Food and Farming. Brussels. https:// ec.europa.eu/agriculture/sites/agriculture/ files/future-of-cap/future_of_food_and_ farming_communication_en.pdf.

Fleury P., Seres C., Dobremez L., Nettier B., Pauthenet Y. (2015). "Flowering Meadows", a result-oriented agri-environmental measure: Technical and value changes in favour of biodiversity. Land Use Policy, $\mathrm{n}^{\circ} 46$, pp. 103-114. https://doi.org/10.1016/ j.landusepol.2015.02.007.

Franks D. J. R., McGloin A. (2007). Joint submissions, output related payments and environmental co-operatives: Can the Dutch experience innovate UK agri-environment policy? Journal of Environmental Planning and Management, $\mathrm{n}^{\circ} 50$, pp. 233-256, https://doi.org/10.1080/09640560601156482.

Frondel M., Lehmann P., Wätzold F. (2012). The impact of information on landowners' participation in voluntary conservation programs - Theoretical considerations and empirical evidence from an agri-environment program in Saxony, Germany. Land Use Policy, $\mathrm{n}^{\circ} 29$, pp. 388-394, https://doi. org/10.1016/j.landusepol.2011.08.003.

Gassiat A., Zahm F. (2013). Améliorer la qualité de l'eau : quelle territorialisation? Exemple des MAE à « enjeu eau ». Économie rurale, $\mathrm{n}^{\circ} 333$, pp. 85-104. https://doi.org/10.4000/ economierurale. 3812 .

Gault J., Pringault J.-M., Riou Y. (2013). Mission de parangonnage (Allemagne, Pays Bas, Autriche, Italie, Angleterre) des mesures agri-environnementales à enjeu eau. Partie 1, CGAAER, $n^{\circ}$ 12079, Ministère de l'Agriculture, de l'Agroalimentaire et de la Forêt, $101 \mathrm{p}$.

Giannakopoulos N., Damianos D. (2002). Farmers' participation in agri-environmental schemes in Greece. British Food Journal, $\mathrm{n}^{\circ}$ 104,pp. 261-273, https://doi.org/10.1108/ 00070700210425705.

Giovanopoulou E., Nastis S. A.,Papanagiotou E. (2011). Modeling farmer participation in agri-environmental nitrate pollution reducing schemes. Ecological Economics, Special Section-Earth System Governance: Accountability and Legitimacy, $\mathrm{n}^{\circ} 70$, pp. 2175-2180, https://doi.org/10.1016/j. ecolecon.2011.06.022.

Herzon I., Birge T., Allen B., Povellato A., Vanni F., Hart K., Radley G., Tucker G., Keenleyside C., $\quad$ Oppermann R., Underwood E., Poux X., Beaufoy G., Pražan J. (2018). Time to look for evidence: Results-based approach to biodiversity conservation on farmland in Europe. Land Use Policy, $\mathrm{n}^{\circ} 71$, pp. 347-354, https://doi. org/10.1016/j.landusepol.2017.12.011.

IEEP (2011). Delivering environmental benefits through entry-level agri-environment schemes in the EU. Projet ENV.B.1/ ETU/2010/0035, $191 \mathrm{p}$.

Jacquet F., Butault J.-P., Guichard L. (2011). An economic analysis of the possibility of reducing pesticides in French field crops. 
Ecological Economics, Special SectionGoverning the Commons: Learning from Field and Laboratory Experiments, $\mathrm{n}^{\circ} 70$, pp. 1638-1648, https://doi.org/10.1016/j. ecolecon.2011.04.003.

Jongeneel R. A.,

Polman N. B. P., Slangen L. H. G. (2008). Why are Dutch farmers going multifunctional? Land Use Policy, $\mathrm{n}^{\circ} 25$, pp. 81-94, https://doi.org/ 10.1016/j.landusepol.2007.03.001.

Keenleyside C., Allen B., Hart K., Menadue H., Stefanova V., Prazan J., Herzon I., Clement T., Povellato A., Maciejczak M., Boatman N. (2011). Delivering environmental benefits through entry-level agrienvironment schemes in the EU, 209 p.

Knop E., Kleijn D., Herzog F., Schmid B. (2006). Effectiveness of the Swiss agrienvironment scheme in promoting biodiversity. Journal of Applied Ecology, $n^{\circ} 43$, pp. 120-127, https://doi.org/10.1111/j.13652664.2005.01113.x.

Kuhfuss L. (2013). Contrats agroenvironnementaux : évaluation et dispositifs innovants en France (thesis). Montpellier 1, $266 \mathrm{p}$.

Kuhfuss L., Jacquet F., Preget R., Thoyer S. (2012). Le dispositif des MAEt pour l'enjeu eau : une fausse bonne idée ? Revue d'études en agriculture et environnement-Review of Agricultural and Environmental Studies, $\mathrm{n}^{\circ}$ 93, pp. 395-422.

Kuhfuss L., Préget R., Thoyer S. (2014). Préférences individuelles et incitations collectives : quels contrats agroenvironnementaux pour la réduction des herbicides par les viticulteurs? Revue d'études en agriculture et environnement, $\mathrm{n}^{\circ}$ 95, pp. 111-143.

Kuhfuss L., Preget R., Thoyer S., Hanley N. (2016). Nudging farmers to enrol land into agri-environmental schemes: the role of a collective bonus. European Review of Agricultural Economics, $\mathrm{n}^{\circ}$ 43, pp. 609-636.

Lastra-Bravo X. B., Hubbard C., Garrod G., Tolón-Becerra A. (2015). What drives farmers' participation in EU agri-environmental schemes? Results from a qualitative metaanalysis. Environmental Science \& Policy, $\mathrm{n}^{\circ}$ 54, pp. 1-9, https://doi.org/10.1016/ j.envsci.2015.06.002.
Latacz-Lohmann U., Breustedt G. (2019). Using choice experiments to improve the design of agri-environmental schemes. European Review of Agricultural Economics, $\mathrm{n}^{\circ}$ 46, pp. 495-528. https://doi.org/10.1093/ erae/jbz020.

Lichtenberg E. (2004). Cost-Responsiveness of Conservation Practice Adoption: A Revealed Preference Approach. Journal of Agricultural and Resource Economics, $\mathrm{n}^{\circ} 29$, pp. 420-435.

Ma S., Swinton S., Lupi F., Jolejole-Foreman C. (2012). Farmers' Willingness to Participate in Payment-for-Environmental-Services Programmes. Journal of Agricultural Economics, $\quad \mathrm{n}^{\circ}$ 63, https://doi.org/10.1111/ j.1477-9552.2012.00358.x.

McGurn P., Moran J. (2013). A National Outcome-based Agri-environment Programme Under Ireland's Rural Development Programme 2014-2020. Report produced for the Heritage Council.

Menozzi D., Fioravanzi M., Donati M. (2015). Farmer's motivation to adopt sustainable agricultural practices. Bio-based and Applied Economics, ${ }^{\circ} 4$, pp. 125-147. https://doi. org/10.13128/BAE-14776.

Mettepenningen E., Beckmann V., Eggers J. (2011). Public transaction costs of agrienvironmental schemes and their determinants-Analysing stakeholders' involvement and perceptions. Ecological Economics, $\mathrm{n}^{\circ} 70$, pp. 641-650, https://doi.org/10.1016/j. ecolecon.2010.10.007.

Ministère de l'Agriculture, de l'Agroalimentaire et de la Forêt (MAAF) (2017). Évaluation ex-post du Programme de Développement Rural Hexagonal (PDRH). Programmation FEADER 2007/2013, tome 2, 169 p.

Murphy G., Hynes S., Murphy E., O’Donoghue C., Green S. (2011). Assessing the compatibility of farmland biodiversity and habitats to the specifications of agri-environmental schemes using a multinomial logit approach. Ecological Economics, $\mathrm{n}^{\circ} 71$, pp. 111-121, https://doi.org/10.1016/j. ecolecon.2011.08.010.

Ouvrard E. (2019). Données chiffrées sur les mesures agro-environnementales et climatiques (MAEC) en Nouvelle-Aquitaine. Campagnes : $\quad$ 2015/2016/2017/2018, 
DRAAF NA / SREAA http://draaf.nouvelleaquitaine.agriculture.gouv.fr/IMG/pdf/ Atlas_dept_MAEC_2019_cle0f5244.pdf.

Peterson J. M. (2011). Buying environmental improvements from farmers who screen on contrac tattributes. AERE Inaugural Summer Conference, Seattle, $17 \mathrm{p}$.

Polman N. B. P., Slangen L. H. G. (2008). Institutional design of agri-environmental contracts in the European Union: the role of trust and social capital. NJAS, Wageningen Journal of Life Sciences, $\mathrm{n}^{\circ} 55$, pp. 413-430, https://doi.org/10.1016/S15735214(08)80029-2.

Pywell R. F., Heard M. S., Bradbury R. B., Hinsley S., Nowakowski M., Walker K. J., Bullock J. M. (2012). Wildlife-friendly farming benefits rare birds, bees and plants. Biology Letters, $\mathrm{n}^{\circ}$ 8, pp. 772-775, https:// doi.org/10.1098/rsbl.2012.0367.

Riley M., Sangster H., Smith H., Chiverrell R., Boyle J. (2018). Will farmers work together for conservation? The potential limits of farmers' cooperation in agrienvironment measures. Land Use Policy, $\mathrm{n}^{\circ} 70$, pp. 635-646, https://doi.org/10.1016/ j.landusepol.2017.10.049.

Schwarz G., Morkvenas Z. (2013). Increasing the effectiveness of agri-environmental measures in Lithuania: guidelines for the practical implementation of resultoriented payments. The Sixth International Scientific Conference Rural Development 2013 Innovations and Sustainability, 28-29 November, Aleksandras Stulginskis University: proceedings, vol. 6, Book 1, pp. 608-614.

Stoneham G., Chaudhri V., Ha A., Strappazzon L. (2003). Auctions for conservation contracts: an empirical examination of Victoria's BushTender trial. Australian Journal of Agricultural and Resource Economics, $\mathrm{n}^{\circ} 47$, pp. 477-500, https:// doi.org/10.1111/j.1467-8489.2003.t01-100224.x.
Thoyer S., Préget R. (2019). Enriching the CAP evaluation toolbox with experimental approaches: introduction to the special issue. European Review of Agricultural Economics, n $^{\circ} 46$, pp. 347-366, https://doi. org/10.1093/erae/jbz024.

Thoyer S., Saïd S. (2007). Mesures agroenvironnementales: quels mécanismes d'allocation? In Doussan I., Dubois J. (dir.), Conservation de la biodiversité et PAC de l'Union européenne, Monde européen et international, Paris, La Documentation française, pp. 121-150, http://prodinra.inra. fr/record/22523.

Unay Gailhard İ., Bojnec Š. (2015). Farm size and participation in agri-environmental measures: Farm-level evidence from Slovenia. Land Use Policy, $\mathrm{n}^{\circ} 46$, pp. 273-282, https://doi.org/10.1016/j.landusepol.2015.03.002.

Urbano G. Vollet D. (2005). L'évaluation du contrat territorial d'exploitation (CTE). Notes et études économiques, $\mathrm{n}^{\circ} 22$, pp. 69-110.

Van Dijk W. F. A., Lokhorst A. M., Berendse F., de Snoo G. R. (2015). Collective agrienvironment schemes: How can regional environmental cooperatives enhance farmers' intentions for agri-environment schemes? Land Use Policy, ${ }^{\circ}$ 42, pp. 759-766, https:// doi.org/10.1016/j.landusepol.2014.10.005.

Vukina T., Zheng X., Marra M., Levy A. (2008). Do farmers value the environment? Evidence from a conservation reserve program auction. International Journal of Industrial Organization, $\mathrm{n}^{\circ} 26$, pp. 1323-1332.

Wezel A., Vincent A., Nitsch H., Schmid O., Dubbert M., Tasser E., Fleury P., Stöckli S., Stolze M., Bogner D. (2018). Farmers' perceptions, preferences, and propositions for result-oriented measures in mountain farming. Land Use Policy, $\mathrm{n}^{\circ} 70$, pp. 117-127. https://doi.org/10.1016/j.landusepol.2017.10.020. 